\title{
Evaluation of Transcranial Magnetic Stimulation Efficiency in Major Depressive Disorder Patients: A Magnetic Resonance Spectroscopy Study
}

\author{
Mehmet Fatih Erbay ${ }^{\bowtie}$, Esra Porgalı Zayman², Lale Gönenir Erbay², and Süheyla Ünal² \\ ${ }^{1}$ Department of Radiology, Inonu University, Malatya, Turkey \\ ${ }^{2}$ Department of Psychiatry, Inonu University, Malatya, Turkey
}

Objective Repetitive transcranial magnetic stimulation (rTMS) is an effective treatment for major depressive disorder (MDD). This study evaluated the antidepressant effect of rTMS and examined how it affected N-asetyl aspartate (NAA), choline (Cho), creatine (Cr), lactate (Lac), myoinositol (mIns), glutamate (Glu), glutathione (GSH), and glutamine (Gln) metabolite levels in the left dorsolateral prefrontal cortex (DLPFC) of MDD patients who were not receiving antidepressant medication.

Methods In total, 18 patients (10 female, 8 male) were evaluated. Each patient underwent $\mathrm{H}$ magnetic resonance spectroscopy (H-MRS) before and within 3 days of completion of TMS therapy. All patients completed 20 sessions of rTMS directed at the left DLPFC over a 2-week period. The Hamilton Depression Scale (HAMD) scores of patients were calculated, and their responses to treatment were assessed within 1-3 days of completion of TMS.

Results We found statistically significant differences in HAMD scores before and after rTMS. Moreover, the peak metabolite ratios of NAA/Cr, GSH/Cr, and Gln/Cr were significantly higher after rTMS compared to those before rTMS.

Conclusion Increased understanding of the mechanism of action of TMS will improve its application and may stimulate development of new-generation therapeutic agents.

Psychiatry Investig 2019;16(10):745-750

Key Words Transcranial magnetic stimulation, Depression, Magnetic resonance spectroscopy, Brain metabolites.

\section{INTRODUCTION}

Repetitive transcranial magnetic stimulation (rTMS) is a noninvasive technique of brain stimulation that is based on the principles of electromagnetic induction to produce an electrical current on the surface of the brain cortex. ${ }^{1}$ It is an efficient treatment option, particularly for patients with major depressive disorder (MDD) who are resistant to antidepressant medications. ${ }^{2,3}$ Previous findings revealed that the antidepressant effect of rTMS results from its administration to a region of the prefrontal cortex. ${ }^{4,5}$ Studies regarding the brain regions for administration have revealed that stimulating the dorsolateral

Received: February 26, 2019 Revised: April 8, 2019

Accepted: July 17, 2019

$\square$ Correspondence: Mehmet Fatih Erbay

Department of Radiology, Inonu University, Malatya 44280, Turkey

Tel: +90 422 3410660-5710, Fax: +90 42234107 87,

E-mail: drfatiherbay@hotmail.com

(a) This is an Open Access article distributed under the terms of the Creative Commons Attribution Non-Commercial License (https://creativecommons.org/licenses/bync/4.0) which permits unrestricted non-commercial use, distribution, and reproduction in any medium, provided the original work is properly cited. prefrontal cortex (DLPFC) is more efficacious. Rapid rTMS administration to the left side has an antidepressant effect, whereas stimulating the right side does not. ${ }^{6,7}$

Very little is known about the neurobiological mechanisms of the antidepressant response to rTMS. Although its effect on cortical circuits is known, it is unclear whether the changes in the excitation-inhibition balance of synaptic networks affect the antidepressant response. ${ }^{8}$ Animal trials have shown that rTMS accelerates serotonin and dopamine turnover. ${ }^{9}$ It reduces 5-hydroxytryptamine (5HT) 2 and beta adrenergic receptors in the frontal cortex while increasing 5HT1A receptors in the frontal cortex and cingulate gyrus. On the other hand, rTMS upregulates $\mathrm{N}$-methyl-D-aspartate receptors in the ventromedial hypothalamus, basolateral amygdala and parietal cortex..$^{10}$

${ }^{1} \mathrm{H}$ Magnetic resonance spectroscopy (H-MRS) is a non-invasive imaging method used to detect endogenous chemical metabolites, including $\mathrm{N}$-acetylaspartate (NAA), choline (Cho), creatine (Cr), lactate (Lac), myoinositol (mIns), glutamate (Glu), glutathione (GSH), and glutamine (Gln). It is possible to yield information about the neurobiological structure of the brain 
using the concentrations of 17 different neurochemical substances determined by H-MRS on 3 Tesla MR systems. ${ }^{11}$ The standard target region for rTMS is the left DLPFC. It has been emphasized in the literature that the same brain locations should be selected for ${ }^{1} \mathrm{H}$-MRS to assess metabolite changes in response to TMS. $^{12} \mathrm{Cr}$ is a reference metabolite that resonates at $3.0 \mathrm{ppm}$ in ${ }^{1} \mathrm{H}$-MRS. As NAA is a neuronal marker, a low NAA/Cr ratio represents neuronal loss and axonal damage. Furthermore, a high $\mathrm{Cho} / \mathrm{Cr}$ ratio reflects synthesis of membranes and cells because Cho exists in the cell membrane.

Several studies have investigated the efficacy of drug treatment, ${ }^{13}$ group therapy, ${ }^{14}$ and mood-regulating medications ${ }^{15}$ by ${ }^{1} \mathrm{H}-\mathrm{MRS}$, but to the best of our knowledge, the therapeutic efficacy of rTMS in the treatment of depression has not been investigated. Initial studies regarding the antidepressant mechanism of TMS revealed an association between frontal perfusion and the response of rTMS. ${ }^{16}$ Subsequent investigations have shown that TMS leads to an augmentation in ion flow via a depolarization wave as well as to several changes in 5HT2 and noradrenergic receptors in neurons. ${ }^{17}$ Some authors have also revealed that TMS acts on monoaminergic systems, such as serotonin, dopamine, gamma-aminobutyric acid and Glu. ${ }^{18,19}$

In this study, we evaluated the antidepressant response of rTMS and how it affects the levels of NAA, Cr, Cho, Gln, GSH, mIns, Glu and Lac metabolites in the left DLPFC of patients with MDD who do not take antidepressant medication.

\section{METHODS}

\section{Participants}

In a power analysis [ $\alpha=0.0051-\beta$ (power): 0.80$]$, the required sample size was calculated as at least 17 patients, so that the average change in brain metabolites in the depressed patients who undergo rTMS should be 6.5 units in ${ }^{1} \mathrm{H}$-MRS. A consort diagram model was used to describe the study patients.

Twenty-three patients who were diagnosed with MDD based on The Diagnostic and Statistical Manual of Mental Disorders, fifth edition (DSM-5), criteria by a psychiatrist using the Structured Clinical Interview for DSM-5 and who did not take any antidepressant medication for at least 3 months were included. Other inclusion criteria were non-smokers, non-users of alcohol or other substances, no suicidal thoughts and/or any previous attempt, and eligible for rTMS treatment. All patients signed written informed consent forms to participate in the study. Exclusion criteria were pregnancy or lactation, diagnosis of a bipolar or psychotic disorder, a clinically significant personality disorder, history of a substance abuse disorder or substance addiction, history of epilepsy or any other neurological illness, history of closed head trauma accompanying loss of consciousness and left-handedness. Each patient underwent
${ }^{1} \mathrm{H}-\mathrm{MRS}$ before and within 3 days of completing TMS therapy. Three patients were excluded due to inappropriate spectra caused by motion artefacts, and two patients were excluded because they did not attend the second spectroscopic imaging after TMS. No side effects were observed during TMS treatment, and all patients completed the treatment. Eighteen patients were evaluated for the analysis. All patients underwent H-MRS and completed the HAMD at the same time within 1-3 days after completion of 2 weeks of TMS treatment to assess treatment responses. This study protocol was approved by the Ethics Committee of Inonu University School of Medicine (IRB authorization number: 2017/05) and conducted in accordance with the ethical principles of the 2013 Declaration of Helsinki and Good Clinical Applications.

\section{rTMS protocol}

All patients completed 20 sessions of rTMS directed to the left DLPFC over a 2-week period using the Neuro-MS/D TMS Therapy System (Neurosoft, Ltd., Ivanova, Russia). Individual sessions consisted of $37.5 \mathrm{~min}$ (3,000 pulses; 30-s duty cycle of $4 \mathrm{~s}$ on and $26 \mathrm{~s}$ off) and $10-\mathrm{Hz}$ excitatory TMS twice a day for 10 days (Monday-Friday over a 2 -week period). The resting motor threshold was defined as the stimulus strength over the thumb area of the motor cortex that produced visually detectable thumb motion. The standardized treatment location was the left DLPFC (middle frontal gyrus). Initial collocation was determined according to the " $5-\mathrm{cm}$ rule" ( $5 \mathrm{~cm}$ forward to the resting motor threshold site along a left superior oblique plane, with a rotation point about the tip of the participant's nose)..$^{20}$

\section{${ }^{1} \mathrm{H}$-MRS neuroimaging procedures}

All patients underwent MRS imaging before and after the rTMS procedure. Whole MRS images were taken at the Inonu University School of Medicine, Radiology Department on a 3T MR device (Magnetom Skyra-Siemens, Erlangen, Germany) using of a 20-channel phase array head coil. The same brain regions were carefully selected to assess the effect of TMS on the metabolite ratios during MRS. ${ }^{12}$ To determine voxel localization and exclude parenchymal lesions, T1-weighted sagittal three-dimensional magnetization-prepared rapid-acquisition gradient echo and T2-weighted fluid-attenuated inversion recovery axial-sagittal images were obtained, respectively. The imaging parameters were as follows: TR: 2,300 ms; TE: 2.98 ms; slice thickness: $1 \mathrm{~mm}$; FOV $256 \mathrm{~mm}$; matrix size: $240 \times 256$. After ruling out pathological lesions on the T2 sequence, thin slice images $(1 \mathrm{~mm})$ at three orthogonal planes (sagittal, axial and coronal) were obtained by multiplanar reconstruction. The borders of the dorsolateral prefrontal lobe were delineated on the coronal plane by tracing a line manually, combining the 
superior frontal sulcus, lateral fissure and outer surface of the cortex in the left cerebral hemisphere at the level of the corpus callosum (genu) (Figure 1). We placed a single $15 \times 20 \times 15 \mathrm{~mm}$

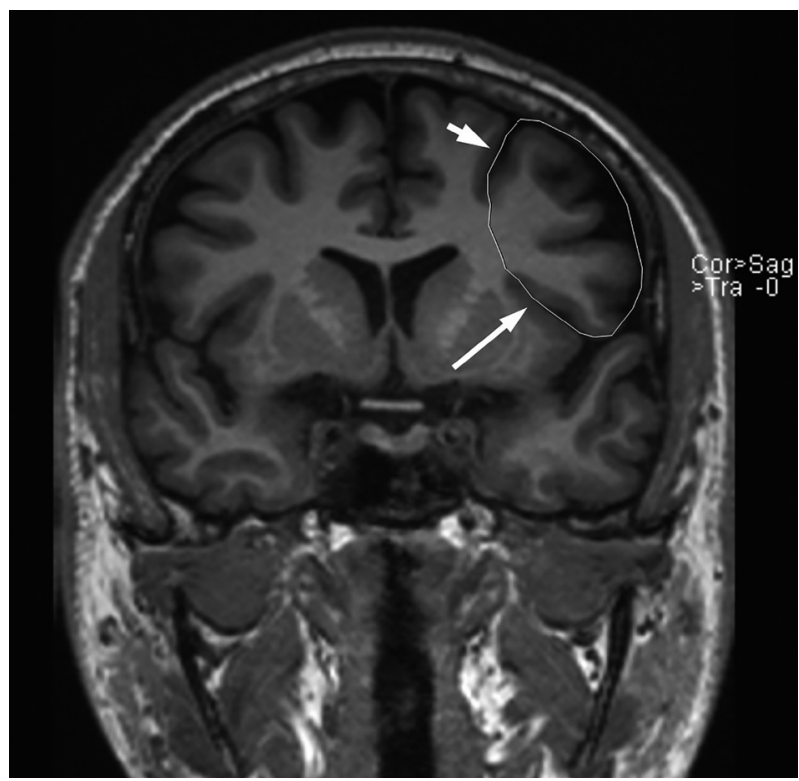

Figure 1. DLPFC segmentation by manually tracing. DLPFC boundaries delineated through a line combining superior frontal sulcus (short arrow), posterior lateral sulcus (long arrow) and outer surface of cortex. DLPFC: dorsolateral prefrontal cortex. volume of interest in a cortical region outside the sulci to avoid cerebrospinal fluid contamination, and single voxel spectroscopy-short echo spectroscopic imaging was performed using a point-resolved spectroscopy sequence (TR: 2000, TE: 30) (Figure 2). The optimal proton signal was obtained by additional "manual shimming" to enhance local magnetic homogeneity in the voxel. Spectroscopic data were transferred to a work station, and peak metabolite ratios were calculated automatically using software (syngo.via-Siemens Healthineers, Erlangen, Germany) after establishing the spectra by proportionating the integral values of the metabolites to that of $\mathrm{Cr}$, which was used as the reference metabolite. ${ }^{21}$

\section{Statistical analysis}

Statistical analyses were performed using the Statistical Program for Social Sciences for Windows, version 17.0, software (SPSS Inc., Chicago, IL, USA). Descriptive statistics for continuous variables are presented as means and standard deviations, and the categorical variables are presented as numbers and percentages. Normality of the data distribution was assessed using the Shapiro-Wilk test. Normally distributed data are summarized as means and standard deviations, and the t-test was used to compare dependent groups. Data not following a normal distribution are summarized as medians and ranges and

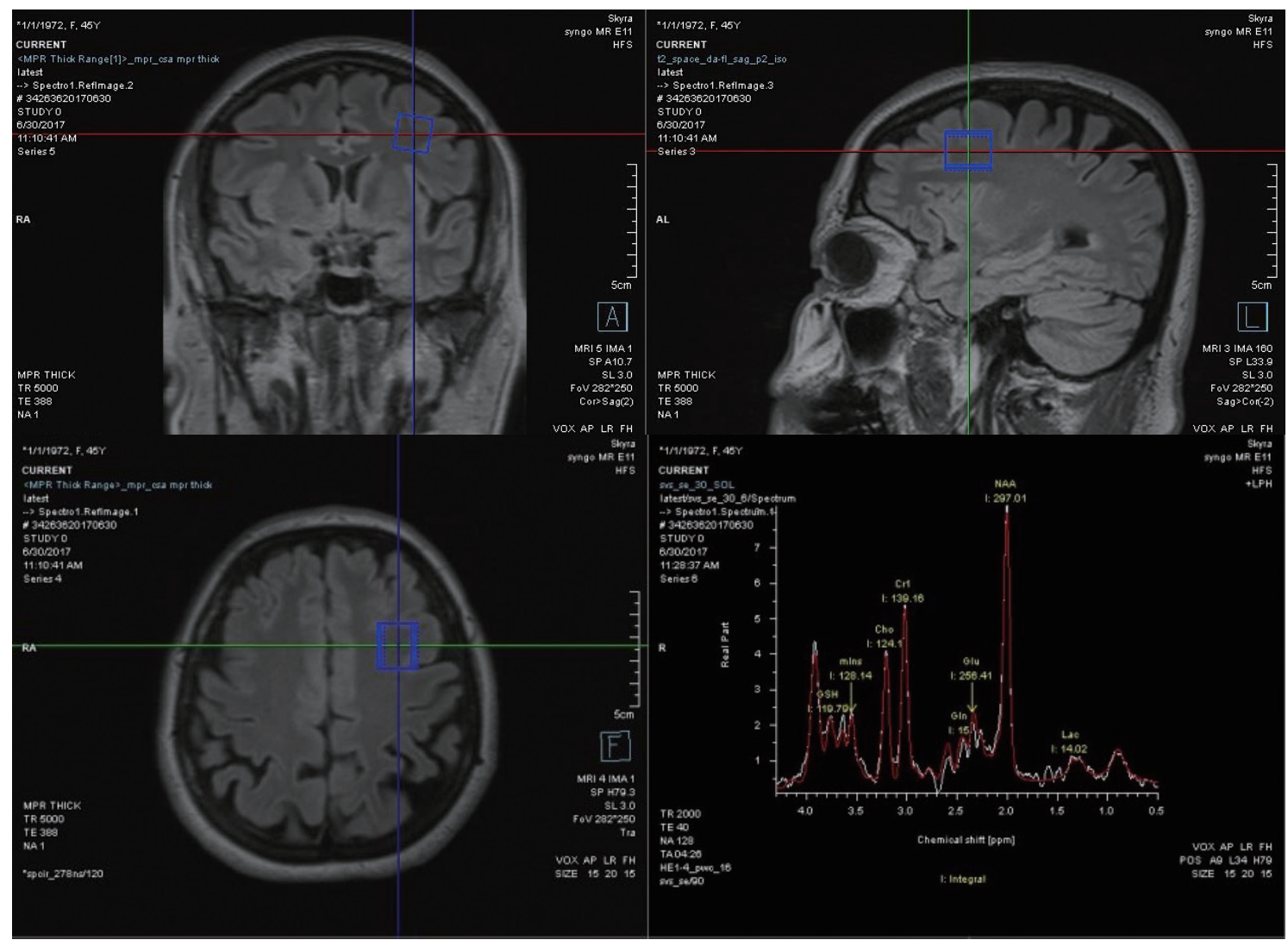

Figure 2. VOI placing into left DLPFC at 3 planes and MR spectrum. VOI: Volume of Interest, DLPFC: Dorsolateral Prefrontal Cortex, MR: magnetic resonance 
were compared using the Wilcoxon test. Spearman's rank correlation test was used to assess the strength of the relationship between the metabolite levels and HAMD scores. A p-value $<$ 0.05 was considered significant.

\section{RESULTS}

The mean age of the patients included in this study was 43.38 \pm 11.14 years. Of the 18 patients, 10 were female $(55.6 \%)$ and 8 were male (44.4\%). Three patients (16.7\%) were additionally diagnosed with an anxiety disorder. A significant difference was observed in the HAMD scores before versus after rTMS $(\mathrm{p}<0.001)$. Moreover, the peak metabolite ratios of $\mathrm{NAA} / \mathrm{Cr}$, $\mathrm{GSH} / \mathrm{Cr}$ and $\mathrm{Gln} / \mathrm{Cr}$ after rTMS were significantly higher compared with those prior to $\mathrm{rTMS}$ ( $\mathrm{p}=0.016, \mathrm{p}=0.040$ and $\mathrm{p}=0.008$, respectively) (Table 1$)$.

The increases in the NAA/Cr, GSH/Cr and $\mathrm{Gln} / \mathrm{Cr}$ ratios were not correlated with the change in the HAMD score after rTMS ( $\mathrm{p}=0.176, \mathrm{p}=0.998$, and $\mathrm{p}=0.649$, respectively).

\section{DISCUSSION}

The main goal of our study was to assess the effect of rTMS on brain chemical metabolites in patients with MDD. Despite the increasing number of studies in the past decade that have investigated the efficacy and action mechanism of rTMS, the antidepressant effect of rTMS is poorly understood.

The most conspicuous findings of our study were the significant increases in peak NAA/Cr, GSH/Cr, and Gln/Cr metabolite ratios and the decrease in the HAMD score after rTMS. The DLPFC is the major region of the prefrontal cortex thought to play an important role in the pathogenesis of MDD and contribute to mood regulation and executive functions. ${ }^{22}$ The standard target region for rTMS is the left DLPFC. It has been em-

Table 1. Comparison of metabolite ratios prior to and after rTMS

\begin{tabular}{lccc}
\hline & $\begin{array}{c}\text { Prior to TMS } \\
\mathrm{N}=18\end{array}$ & $\begin{array}{c}\text { After TMS } \\
\mathrm{N}=18\end{array}$ & p value \\
\hline $\mathrm{NAA} / \mathrm{Cr}$ & $2.2 \pm 0.25$ & $2.31 \pm 0.27$ & 0.016 \\
$\mathrm{Cho} / \mathrm{Cr}$ & $0.85 \pm 0.13$ & $0.81 \pm 0.1$ & 0.399 \\
$\mathrm{mIns} / \mathrm{Cr}$ & $1.33 \pm 0.37$ & $1.41 \pm 0.41$ & 0.503 \\
$\mathrm{Glu} / \mathrm{Cr}$ & $1.89 \pm 0.74$ & $2.03 \pm 0.49$ & 0.467 \\
$\mathrm{GSH} / \mathrm{Cr}$ & $1.17 \pm 0.31$ & $1.4 \pm 0.37$ & 0.040 \\
$\mathrm{Lac} / \mathrm{Cr}$ & $0.2(0-1.49)$ & $0.53(0.05-5.46)$ & 0.112 \\
$\mathrm{Gln} / \mathrm{Cr}$ & $0.17(0-4.17)$ & $0.62(0.14-9.36)$ & 0.008 \\
HAMD & $23.06 \pm 5.97$ & $11.5 \pm 2.5$ & $<0.001$ \\
\hline
\end{tabular}

rTMS: repetetive transcranial magnetic stimulation, N: number of samples, NAA: N-asetyl aspartate, Cho: choline, Cr: creatine, Lac: lactate, mIns: myoinositol, Glu: glutamate, GSH: glutathione, Gln: glutamine, HAMD: Hamilton Depression Scale phasized in the literature that the same brain locations should be selected for ${ }^{1} \mathrm{H}$-MRS to assess the metabolite changes in response to rTMS. ${ }^{20}$ Therefore, we measured the peak metabolite ratios only in the left DLPFC by ${ }^{1} \mathrm{H}-\mathrm{MRS}$. MRS studies on depression have revealed metabolite changes in several brain regions, such as the prefrontal cortex, ${ }^{23}$ anterior cingulate cortex, ${ }^{24}$ occipital cortex,,$^{25}$ cerebellum, ${ }^{24}$ right caudate nucleus and putamen $^{26}$ and hippocampus. ${ }^{27}$

NAA exists in neurons but not astrocytes and is responsible for neuronal integrity. ${ }^{28}$ Wang et al. ${ }^{23}$ reported lower NAA/Cr ratios in the right and left DLPFC of patients with moderate and severe MDD, compared with healthy subjects, in a MRS study. Another study also reported a lower NAA/Cr ratio in the prefrontal cortex of patients with MDD than in healthy controls. ${ }^{29}$ The decline in the NAA/Cr ratio in the prefrontal cortex has been attributed to reduced neuronal density or neuronal dysfunction. Several studies have revealed that the decrease in the NAA level might play a role in the etiology of psychiatric diseases. Furthermore, some researchers have focused on the therapeutic efficacy of NAA. A study investigating the outcomes of lithium treatment revealed that lithium administered for 4 weeks increases NAA concentrations in all brain regions, including the frontal, temporal, parietal and occipital lobes. This result was attributed to the neuroprotective effect of lithium. ${ }^{30}$ In contrast, after pharmacological treatment, increases in hippocampal NAA and Cho metabolite levels, ${ }^{12}$ the basal ganglia $\mathrm{Cho/Cr}$ ratio, ${ }^{31}$ levels of NAA, Glu, Gln and mIns in the anterior cingulate cortex, ${ }^{32}$ the NAA/Cr ratio in the medial prefrontal corte $^{33}$ and the $\mathrm{Cho} / \mathrm{Cr}$ ratio in the ventral prefrontal corte $\mathrm{x}^{34}$ were also recorded. These metabolite changes were accepted as indicators of the neuronal regenerative effect due to antidepressant medication. ${ }^{35}$

Glu is the leading excitatory neurotransmitter in the brain, and its homeostasis is modulated by the $\mathrm{Glu} / \mathrm{Gln}$ cycle between neurons and astrocytes. Evidence shows that hypoactivity and lower levels of Glu/Gln in the cortex of patients with MDD are closely associated with a lower glutamatergic signaling frequency. ${ }^{36}$ One study revealed that direct infusion of exogenous Gln into the medial prefrontal cortex results in an improvement in depressive-like behaviors in mice. ${ }^{37}$ Son et al. ${ }^{36}$ suggested that depressive behaviors due to chronic stress in mice are associated with hypoactive neurons in the medial prefrontal cortex, and that exogenous Gln can be used to augment glutamatergic neurotransmission as an alternative antidepressant. Michael et al. ${ }^{38}$ reported lower Glu/Gln levels prior to unipolar electroconvulsive therapy in the left DLPFC of patients with severe MDD compared with the control group and showed normalized levels after electroconvulsive therapy without any significant difference between patients and controls.

GSH is an antioxidant that protects cells and their contents 
against oxidative stress to maintain tissue functions. Therefore, it is a sensitive endogenous determining factor of oxidative stress. ${ }^{39}$ Wide-ranging clinical evidence reveals that the inflammatory response and oxidative damage are intensified in patients with MDD. ${ }^{40,41}$ Furthermore, some studies have reported that long-term antidepressant therapy (12-14 weeks) reverses the increased oxidative stress in patients with MDD. ${ }^{40}$ Nevertheless, it remains unclear whether the decrease in GSH is an etiological factor in MDD or an outcome of the disease itself. However, the therapeutic usage of agents such as acetylcysteine for adult neuropsychiatric disorders is still a subject under investigation. ${ }^{42,43}$

In our study, the increases in the peak metabolite NAA/Cr, $\mathrm{GSH} / \mathrm{Cr}$ and $\mathrm{Gln} / \mathrm{Cr}$ ratios after rTMS treatment suggest that the therapeutic effect of TMS result from these metabolites, which are thought to play a role in the pathogenesis of depression. However, more evidence is needed to conclude that TMS has a regenerative effect on neuronal damage via these metabolites. Additionally, it remains unclear how TMS leads to increased levels of the metabolites.

One of the limitations of our study was the small number of patients. Further studies involving a larger sample size will contribute to a better understanding of how TMS works in depression treatment. Selecting patients who have not taken medications and minimizing variables that may affect the results by using broad exclusion criteria will render our findings more valuable. Three MDD patients included in the study had an additional diagnosis of anxiety disorder, Although this partially reduced the homogeneity of patients' diagnostic profiles, depression is often accompanied by an anxiety disorder.

Another limitation was the lack of attention to the long-term effects of TMS and the lack of a placebo control group, which would have improved our ability to distinguish the effects of TMS from those of other interventions.

In conclusion, studies investigating the antidepressant mechanism of TMS are of great importance in two respects. First, they increase evidence on the abnormal circuits that are affected, and second, the findings will help determine whether individualizing depression treatment by determining relevant indicators predicting the treatment response will lead to better clinical outcomes. Understanding the mechanism of action of TMS will improve its application and perhaps stimulate the development of a new generation of therapeutic agents in the future.

\section{Acknowledgments}

This project was supported by the Inonu University Scientific Research Projects Unit (project ID: 921; project code: TSA-2018-921).

\section{Conflicts of Interest}

The authors have no potential conflicts of interest to disclose.

\section{Author Contributions}

Conceptualization: Mehmet Fatih Erbay, Esra Porgalı Zayman. Data curation: Lale Gönenir Erbay. Formal analysis: Lale Gönenir Erbay. Investigation: Esra Porgalı Zayman. Methodology: Mehmet Fatih Erbay, Lale Gönenir Erbay. Project administration: Lale Gönenir Erbay. Resources: Esra Porgalı Zayman. Software: Mehmet Fatih Erbay. Supervision: Süheyla Ünal. Validation: Lale Gönenir Erbay. Visualization: Mehmet Fatih Erbay, Lale Gönenir Erbay. Writing_original draft: Mehmet Fatih Erbay, Lale Gönenir Erbay. Writing_-review \& editing: Mehmet Fatih Erbay, Esra Porgalı Zayman, Lale Gönenir Erbay.

\section{ORCID iDs}

Mehmet Fatih Erbay https://orcid.org/0000-0002-1596-3147

Esra Porgalı Zayman https://orcid.org/0000-0003-1551-6782

Lale Gönenir Erbay https://orcid.org/0000-0002-9969-3016

Süheyla Ünal https://orcid.org/0000-0002-1442-3093

\section{REFERENCES}

1. Wagner T, Rushmore J, Eden U, Valero-Cabre A. Biophysical foundations underlying TMS: setting the stage for an effective use of neurostimulation in the cognitive neurosciences. Cortex 2009;45:1025-1034.

2. George MS, Lisanby SH, Avery D, McDonald WM, Durkalski V, Pavlicova M, et al. Daily left prefrontal transcranial magnetic stimulation therapy for major depressive disorder: a sham-controlled randomized trial. Arch Gen Psychiatry 2010;67:507-516.

3. Blumberger DM, Vila-Rodriguez F, Thorpe KE, Feffer K, Noda Y, Giacobbe $P$, et al. Effectiveness of theta burst versus high-frequency repetitive transcranial magnetic stimulation in patients with depression (THREE-D): a randomised non-inferiority trial. Lancet 2018;391:16831692.

4. Pascual-Leone A, Catalá MD, Pascual-Leone Pascual AÇ. Lateralized effect of rapid-rate rTMS of the prefrontal cortex on mood. Neurology 1996;46:499-502.

5. George MS. Transcranial magnetic stimulation for the treatment of depression. Expert Rev Neurother 2010;10:1761-1772.

6. Pascual-Leoné A, Rubio B, Pallado F, Catala MD. Rapid rate rTMS of left dorsolateral cortex in drug resistant depression. Lancet 1996;347: 233-237.

7. Yadollahpour A, Hosseini SA, Shakeri A. rTMS for the treatment of depression: a comprehensive review of effective protocols on right DLPFC. Int J Ment Health Addict 2016;14:539-549.

8. Rodriguez-Martin JL, Barbanoj JM, Schlaepfer TE, Clos SSC, Pérez V, Kulisevsky J, et al. Transcranial magnetic stimulation for treating depression. Cochrane Database Syst Rev 2002;(2):CD003493.

9. Fitzgerald PB, Daskalakis ZJ. The effects of repetitive transcranial magnetic stimulation in the treatment of depression. Expert Rev Med Devices 2011;8:85-95.

10. Zangen A, Hyodo K. Transcranial magnetic stimulation induces increases in extracellular levels of dopamine and glutamate in the nucleus accumbens. Neuroreport 2002;13:2401-2405.

11. Tkac I, Öz G, Adriany G, Ugurbil K, Gruetter R. In vivo $1 \mathrm{H}$ NMR spectroscopy of the human brain at high magnetic fields: Metabolite quantification at $4 \mathrm{~T}$ vs. 7T. Mag Reson Med 2009;62:868-879.

12. Hone-Blanchet A, Salas RE, Celnik P, Kalloo A, Schar M, Puts NA, et al. Co-registration of magnetic resonance spectroscopy and transcranial magnetic stimulation. J Neurosci Methods 2015;242:52-57.

13. Block W, Traber F, von Widdern O, Metten M, Schild H, Maier W, et al. Proton MR spectroscopy of the hippocampus at $3 \mathrm{~T}$ in patients with unipolar major depressive disorder: correlates and predictors of treatment response. Int J Neuropsychopharmacol 2009;12:415-422.

14. Oğuzhanoğlu NK, Varma GS, Karadağ F, Tümkaya S, Efe M, Kıroğlu Y. Prefrontal cortex neurochemical metabolite levels in major depression and the effects of treatment: An 1 H MRS study. Turk J Psychiatry 
2013;25:75-83.

15. Cumurcu BE, Karlıdağ R, Saraç K, Ünal S, Özcan C. Evaluation of neuroprotective effects of lithium and olanzapine on brain tissue in bipolar affective disorder: a comperative study with MR spectroscopy. J Anatol Psychiatry 2004;5:197-205.

16. Speer AM, Kimbrell TA, Wassermann EM, D Repella J, Willis MW, Herscovitch P, et al. Opposite effects of high and low frequency rTMS on regional brain activity in depressed patients. Biol Psychiatry 2000; 48:1133-1141.

17. Padberg F, Zwanzger P, Keck ME, Kathmann N, Mikhaiel P, Ella R, et al. Repetitive transcranial magnetic stimulation (rTMS) in major depression: Relation between efficacy and stimulation intensity. Neuropsychopharmacology 2002;27:638-645.

18. Salomons TV, Dunlop K, Kennedy SH, Flint A, Geraci J, Giacobbe P, et al. Resting-state corticothalamic-striatal connectivity predicts response to dorsomedial prefrontal rTMS in major depressive disorder. Neuropsychopharmacology 2014;39:488-498.

19. Dubin MJ, Mao X, Banerjee S, Goodman Z, Lapidus KA, Kang G, et al. Elevated prefrontal cortex GABA in patients with major depressive disorder after TMS treatment measured with proton magnetic resonance spectroscopy. J Psychiatry Neurosci 2016;41:E37-E45.

20. Herwig U, Satrapi P, Schonfeldt-Lecuona C. Using the international 10-20 EEG system for positioning of transcranial magnetic stimulation. Brain Topogr 2003;16:95-99.

21. Angelie E, Bonmartin A, Boudraa A, Gonnaud PR, Mallet JJ, SappeyMariner D. Regional differences and metabolic changes in normal aging of the human brain: Proton MR spectroscopic imaging study. Am J Neuroradiol 2001;22:119-127.

22. Koenigs M, Grafman J. The functional neuroanatomy of depression: distinct roles for ventromedial and dorsolateral prefrontal cortex. Behav Brain Res 2009;201:239-243.

23. Wang Y, Jia Y, Xu G, Ling X, Liu S, Huang L. Frontal white matter biochemical abnormalities in first-episode, treatment-naive patients with major depressive 61 disorder: a proton magnetic resonance spectroscopy study. J Affect Disord 2012;136:620-626.

24. Chen LP, Dai HY, Dai ZZ, Xu CT, Wu RH. Anterior cingulate cortex and cerebellar hemisphere neurometabolite changes in depression treatment: A H magnetic resonance spectroscopy study. Psychiatry Clin Neurosci 2014;68:357-364.

25. Abdallah CG, Niciu MJ, Fenton LR, Fasula MK, Jiang L, Black A, et al. Decreased occipital cortical glutamate levels in response to successful cognitive behavioral therapy and pharmaco therapy for major depressive disorder. Psychother Psychosom 2014;83:289-307.

26. Vythilingam M, Charles HC, Tupler LA, Blitchington T, Kelly L, Krishnan KR. Focal and lateralized subcortical abnormalities in unipolar major depressive disorder: an automated multivoxel proton magnetic resonance spectroscopy study. Biol Psychiatry 2003;54:744-750.

27. De Diego-Adelino J, Portella MJ, Gomez-Anson B, Lopez-Moruelo O, SerraBlasco M, Vives Y, et al. Hippocampal abnormalities of glutamate/glutamine, $\mathrm{N}$-acetylaspartate and choline in patients with depression are related to a stillness burden. Psychiatry Neurosci 2013;38: 107-116.

28. Wu WE, Gass A, Glodzik L, Babb JS, Hirsch J, Sollberger M, et al. Whole brain $\mathrm{N}$-acetylaspartate concentration is conserved throughout normal aging. Neurobiol Aging 2012;33:2440-2447.
29. Jia Y, Zhong S, Wang Y, Liu T, Liao X, Huang L. The correlation between biochemical abnormalities in frontal white matter, hippocampus and serum thyroid 76 hormone levels in first-episode patients with major depressive disorder. J Affect Disord 2015;180:162-169.

30. Moore GJ, Bebchuk JM, Hasanat K, Chen G, Seraji-Bozorgzad N, Wilds IB, et al. Lithium increases N-acetyl-aspartate in the human brain: in vivo evidence in support of bcl-2's neurotrophic effects? Biol Psychiatry 2000;48:1-8.

31. Sonawalla SB, Renshaw PF, Moore CM, Alpert JE, Nierenberg AA, Rosenbaum JF, et al. Compounds containing cytosolic choline in the basal ganglia: A potential biological marker of true drug response to fluoxetine. Am J Psychiatry 1999;156:1638-1640.

32. Auer DP, Pütz B, Kraft E, Lipinski B, Schill J, Holsboer F. Reduced glutamate in the anterior cingulate cortex in depression: an in vivo proton magnetic resonance spectroscopy study. Biol Psychiatry 2000;47:305313.

33. Gönül AS, Kitis O, Ozan E, Akdeniz F, Eker C, Eker OD, et al. The effect of antidepressant treatment on $\mathrm{N}$-acetyl aspartate levels of medial frontal cortex in drug free depressed patients. Prog Neuropsychopharmacol Biol Psychiatry 2006;30:120-125.

34. Zhang Y, Han Y, Wang Y, Zhang Y, Li L, Jin E, et al. A MRS study of metabolic alterations in the frontal white matter of major depressive disorder patients with the treatment of SSRIs. BMC Psychiatry 2015; 15:99.

35. Herman-Sucharska I, Werewka-Maczuga A, Urbanik A, Rachel W, Siwek M, Dudek D, et al. Usefulness of proton MR spectroscopy for determining diagnosis and treatment outcome in unipolar depressive disorder. Przegl Lek 2010;67:243-246.

36. Son H, Baek JH, Go BS, Jung DH, Sontakke SB, Chung HJ, et al. Glutamine has antidepressive effects through increments of glutamate and glutamine levels and glutamatergic activity in the medial prefrontal cortex. Neuropharmacology 2018;25:143-152.

37. Lee Y, Son H, Kim G, Kim S, Lee DH, Roh GS, et al. Glutamine deficiency in the prefrontal cortex increases depressive-like behaviours in male mice. J Psychiatry Neurosci 2013;38:183-191.

38. Michael N, Erfurth A, Ohrmann P, Arolt V, Heindel W, Pfleiderer B. Metabolic changes within the left dorsolateral prefrontal cortex occurring with electroconvulsive therapy in patients with treatment resistant unipolar depression. Psychol Med 2003;33:1277-1284.

39. Dringen R. Metabolism and functions of glutathione in brain. Prog Neurobiol 2000;62:649-671.

40. Behr GA, Moreira JC, Frey BN. Preclinical and clinical evidence of antioxidant effects of antidepressant agents: implications for the pathophysiology of major depressive disorder. Oxid Med Cell Longev 2012; 2012:609421.

41. Bilici M, Efe H, Köroğlu MA, Uydu HA, Bekaroğlu M, Değer O. Antioxidative enzyme activities and lipid peroxidation in major depression: alterations by antidepressant treatments. J Affect Disord 2001;64:43-51.

42. Magalhães PV, Dean OM, Bush AI, Copolov DL, Malhi GS, Kohlmann K, et al. N-acetyl cysteine add-on treatment for bipolar II disorder: a subgroup analysis of a randomized placebo-controlled trial. J Affect Disord 2011;129:317-320.

43. Deepmala, Slattery J, Kumar N, Delhey L, Berk M, Dean O, et al. Clinical trials of $\mathrm{N}$-acetylcysteine in psychiatry and neurology: A systematic review. Neurosci Biobehav Rev 2015;55:294-321. 